(Aus der zoologischen Station zu Arcachon.)

\title{
Elektropathologische Untersuchungen.
}

\author{
II. \\ Zur Elektropathologie der marklosen Kephalopodennerven. \\ Von
}

Prof. H. Hornttau.

(Mit 4 Textfiguren.)

In einer grösseren Untersuchung von mir und Fröhlich ${ }^{1}$ ) sind die Veränderungen, welche die Erregungswelle in Gestalt ihres elektrischen Ausdrucks, des Aktionsstroms, unter der Einwirkung schädigender Agentien, insbesondere der Narkose und der Sauerstoffentziehung, erfährt, genauer am markhaltigen Froschnerven untersucht worden. Die Hauptergebnisse betrafen 1. das [von mir an anderer Stelle ${ }^{2}$ ) für sich besprochene] sogen. Lokalisationsgesetz: Lokalisation der Veränderungen des zeitlichen Verlaufs auf den Bereich der Wirkung des chemischen oder physikalischen Agens, 2. das Dekrement der Erregungswelle, seine Abhängigkeit von der Reizstärke und Länge der geschädigten Strecke; endlich 3. die Reihenfolge der Veränderungen mit zunehmender Dauer der schädigenden Einwirkung, die sich in hohem Masse derjenigen der Veränderungen der Muskelzuckung im Laufe der Frmüdung analog erwies. In der Tat hatte ich ja bereits früher ${ }^{3}$ ) die Verlängerung des absteigenden Schenkels des Aktionsstroms beim tetanisierten Froschnerven konstatiert, und $\mathrm{Fröhlich}$ ) gelang es, Ermüdung des markhaltigen Froschnerven am myographischen Erfolge zum erstenmal bestimmt nachzuweisen dureh die Kombination von Narkose mit starker und frequenter Reizung.

1) Dieses Archiv Bd. 105 S. 444. 1904.

2) Zeitschr. f. allgem. Physiol. Bd. 4 S. 289.1904.

3) Dieses Archiv Bd. 84 S. 309. 1901.

4) Zeitschr. f. allgem. Physiol. Bd. 3 S. 469. 1904.

E. Pflüger, Arehiv für Physiologie. Bd. 107. 
Für den marklosen Olfactorius des Hechtes hatten Miss Sowton und besonders S. Garten in seiner grossen Arbeit bereits vorher gezeigt, dass Ermüdung durch wiederholte Reizung an dem Verhalten des Aktionsstroms sehr leicht zu konstatieren ist. Ferner hatte Garten bei vorübergehender Narkose durch Kohlensäure dieselbe Reihenfolge der Veränderungen des Aktionsstroms am Hechtolfactorius registrieren können, wie wir sie später auch am Froschischiadicus gefunden haben. Den Äther bat endlich Garten dazu benutzt, um den als physiologisch bezeichneten Anteil der elektrotonischen Ströme des Hechtolfactorius von einem anderen, von Biedermann, Hering und ihm als "physikalischer Elektrotonus" bezeichneten Anteil zu trennen, ähnlich wie dies bereits Biedermann getan hatte ${ }^{1}$ ).

Ein zweckmässiges Objekt für die Untersuchungen der elektrischen Erscheinungen bilden die marklosen Mantelnerven grosser

1) Beim Hechtolfactorius sind, wie Garten findet, beide extrapolaren elektrotonischen Ströme deutlich ausgesprochen, der anelektrotonische stärker als der katelektrotouische; bei der kapillarelektrometrischen Untersuchung des zeitlichen Verlaufs ihrer Entwicklung liess sich, wenn der polarisierende Strom stark genug war, ein plötzlich auftretender Anteil und ein langsamer sich entwickelnder konstatieren: besonders verzögert ist die Entwickiung dieses letzteren bei starker Ablühlung, welche ja auch den Verlauf des Aktionsstromes verzögert: „nachhinkender Elektrotonus". Durch Ätherisierung wird dieser langsam sich entwickelnde Anteil aufgehoben, während der andere Anteil bleibt; die Richtung dieses Anteils hängt übrigens von der Lage der ableitenden Elektroden am Nerven ab, womit bewiesen wird, dass es sich um Stromschleifen handelt, wie sie auch an dem Hering'schen Modell aus besser leitendem Kern und schlechter leitender Hülle zu erhalten sind. Diesen Erscheinungen nun die Bezeichnung des "physikalischen Elektrotonus" zuzugestehen, hat wohl nie in der Absicht des Schöpfers der polarisatorischen Kernleitertheorie des Elektrotonus, L. Hermann, gelegen; heutzutage, wo dieselbe durch die Vorstellung einer "Grenzschicht" mit den Eigenschaften einer semipermeablen Membran ersetzt werden muss, ist eine Unterscheidung von physikalischem und physiologischem Elektrotonus erst recht überflüssig. Übrigens glaube ich nicht, dass, wie Garten meint, der "nachhinkende" (physiologische) Elektrotonus der Erklärung durch die "Grenzschichttheorie" irgendwelche Schwierigkeiten macht. Im übrigen habe ich nach meiner Erklärung in diesem Archiv Bd. 105 S. 443 keine Veranlassung, auf diese theoretischen Fragen nochmals einzugehen, und habe darum auch auf weitere Versuche über die zeitliche Entwicklung des Elektrotonus verzichtet, welche doch sicher nichts anderes ergeben würden als die aus dem ausgezeichneten Kurvenmaterial Garten's sich ergebenden Tatsachen. 
Kephal opoden: Die früher von S. Fuchs ${ }^{1}$ ), $\operatorname{mir}^{2}$ ) und Mendels$\operatorname{soh} n^{3}$ ) angestellten Untersuchungen hatten deutlich gezeigt, dass diese in bezug auf Fortpflanzungsgeschwindigkeit und Länge (Zeitdauer) der Erregungswelle sowie Stärke der extrapolaren elektrotonischen Ströme etwa in der Mitte zwischen den markhaltigen Froschnerven und den ganz myelinarmen Nerven - Hechtolfactorius, Muschelnerven u. a. - rangieren. Es erschien deshalb von Interesse, an diesen Objekten gleichfalls die Wirkungen ermüdender Reizung, der Narkose und Erstickung einer summarischen Prüfung zu unterwerfen, mit Rücksicht auf etwaige Unterschiede gegenüber dem Verhalten der in der Reihe höher und tiefer stehender Objekte.

Eine Reihe hierhergehöriger Versuche habe ich in der zoologischen Station zu Arcachon (Südfrankreich, Département Gironde) angestellt. Diese, eine Dépendance der Universität Bordeaux, im wesentlichen von der Société scientifique d'Arcachon erhalten, bietet durch ihre Lage am Strande des austern- und fischreichen Beckens von Arcachon und durch die Einrichtung ihrer Laboratorien vorzügliche Gelegenheit zur Anstellung jeder Art von zoologischen und physiologischen Arbeiten an Seetieren; ich bin ihrem Vorstande, Herrn Professor F. Jolyet, für die gastfreundliche Aufnahme und stete Unterstützung mit allen möglichen Arbeitsbedürfnissen zu allergrösstem Danke verpflichtet.

Von der aus teils mitgebrachten, teils der Station und dem physiologischen Laboratorium der Universität Bordeaux entlehnten Apparaten zusammengestellten instrumentellen Einrichtung bildeten die Hauptbestandteile ein vorzuggliches, hochempfindliches Drehspulgalvanometer von Edelmann in München, von dessen Ablenkung bei $1 \mathrm{~m}$ Skalenabstand jedes Millimeter $4 \cdot 10^{-10}$ Ampère entspricht, das Hermann'sche Rheotom sowie eine improvisierte Einrichtung zur photographischen Registrierung der Ausschläge desselben Kapillarelektrometers, welches zur Aufnahme der Mehrzahl der in der Elektropathologie des Froschischiadicus ${ }^{4}$ ) veröffentlichten Kurven gedient hatte. Als Lichtquelle für die Projektion des letzteren

1) Sitzungsber. der Wiener Akad., math.-physiol. Klasse, Bd. 103 Abt. 3 S. 207.1894.

2) Dieses Archiv Bd. 66 S. 285.1897.

3) Compt. rend, t. 132 p. 1507. 1901.

4) Siehe dieses Archiv Bd. 105 S. 451. 
mussten, da elektrische Starkstromquellen fehlten, die durch einen Heliostaten in den verdunkelten Arbeitsraum reflektierten Sonnenstrahlen dienen. Bei der schon vorgerückten Jahreszeit machte dieses Beschränkung der Registrierversuche auf die mittleren Tagesstunden nötig, während welcher Erschütterung des Apparates durch die in der Station und ihrer Umgebung herrschende. Unruhe nicht immer zu vermeiden war; dazu kam noch die aus den früheren. Veröffentlichungen von $\mathrm{Fuchs}$ und mir bekannte, im Vergleich zu: derjenigen des Ischiadicus viel kleineren Intensität der phasischen Aktionsströme, für welche die Empfindlichkeit des Elektrometers die äusserste Grenze bildete. Es ist daher das zur Verfügung stehende Kapillarelektrometer-Kurvenmaterial ziemlich mangelhaft; indessen hinzugefügt zu einer Reihe von Rheotomversuchen, dient es doch zur Sicherung der im folgenden mitzuteilenden Ergebnisse.

An eine myographische Untersuchung der Wirkung schädigender Agentien auf den Kephalopodennerven war natürlich von vornherein $\mathrm{nich} \mathrm{t} \mathrm{zu}$ de $\mathrm{nken}$, da das seinerzeit von v. Uexküll benutzte „Nervmuskelpräparat", welches aus dem Mantelnerven nebst Stellarganglion, dessen Nervenausstrahlung und einem Teil der Mantelmuskulatur besteht, erstens prinzipiell von einem gewöhnlichen Froschmuskelpräparat gänzlich verschieden ist, zweitens aber, wie ja schon Uexküll fand, viel zu schnell, wohl durch Absterben hauptsächlich der Ganglienzellen und motorischen Nervenendigungen, seine Erregbarkeit verliert. Man musste sich also auf die Untersuchung der elektrischen Erscheinungen beschränken.

Zur Verfügung standen teilweise enorm grosse und kräftige Exemplare von Octopus vulgaris und macropus, von denen ich schon seinerzeit erwähnte, dass die Mantelnerven das beste Objekt für in Rede stehende Zwecke darstellen. Die verschiedenen Arten von Eledone sind in jenen Meeresteilen selten.

Eine ganze Reihe von Erscheinungen sind nun dazu angetan, Untersuchungen wie die vorliegende gerade am Kephalopodennerven ausserordentlich zu erschweren. Manche Präparate sterben sehr schnell ab, insbesondere an warmen Tagen oder bei hoher Zimmertemperatur: hier kann es unmöglich werden, die Wiedererholung von der Narkose oder gar von der Erstickung zu beobachten. Immerhin fand ich auch Nerven von besonders gut ernährten Tieren, welche noch 12 bis 24 Stunden nach dem Ausschneiden, inzwischen in Seewasser aufbewahrt, oder auch in der kühl gehaltenen Feucht- 
kammer den Elektroden aufliegend, deutliche negative Schwankung gaben.

Die absolute Erregbarkeit durch Induktionsströme ist geringer als beim Froschnerven. Es kann so nötig werden, Rollenabstände des Schlitteninduktoriums zu wählen, bei welchen Einmischung des Elektrotonus sowie unipolare Mitreizung ausserhalb der Reizelektrodenstrecke gelegener Nervenstellen unvermeidlich sind : letzteres ist für Versuche über die Lokalisation von Veränderungen der Erregungswelle, überhaupt für alle Untersuchungen ihres zeitlichen Verlạufes, unzulässig.

Trotz alledem konnten folgende Tatsachen sicher festgestellt werden:

In bezug auf Erıü $\mathrm{db}$ arkeit steht der Mantelnerv von Octopus viel näher dem Froschischiadicus als dem Hechtolfactorius: wiederholte langdauernde und kräftige tetanisierende Reizung setzt die Grösse der integralen negativen Schwankung des Demarkationsstroms nicht merklich herab; im Gegenteil, sie erscheint unmerkbar nach der Tetanisation etwas vergrössert, so wie das für den Froschnerven schon $\mathrm{W}$ aller gefunden hat. Beispiel:

Die zwei zusammengelegten Mantelnerven eines grossen Octopus; Entfernung von der Reizelektrode bis zur ableitenden Längsschnittelektrode $r l=15 \mathrm{~mm}$ von der Längsschnittelektrode zur Querschnittelektrode $l q=12 \mathrm{~mm}$. Demarkationsstrom +500 Skalenteile. Schwache Tetanisation mit $270 \mathrm{~mm}$ Rollenabstand.

$11^{\mathrm{h}} 50^{\prime}$. Ablenkung -24 .

11 h 51 '. $\quad$. $\quad-23$.

11 h $52^{\prime}$. $\quad \Rightarrow \quad-23$.

Von $11^{\mathrm{h}} 53^{\prime}$ bis $11^{\mathrm{h}} 54^{\prime}$ zwei Minuten lange Tetanisation (Rollenabst. $170 \mathrm{~mm}$ ). 11 h $55^{\prime}$ (R.-A. $260 \mathrm{~mm}$, wie oben). Ablenkung -27 .

$11 \mathrm{~h} 56$ '. Ablenkung -26 .

11 l $57^{\prime}$. $\quad, \quad-24$.

Pause.

1 h 17 '. Ablenkung -24 .

Diese Vergrösserung rührte, wie die Rheotom- und Kapillarelektrometeruntersuchung zeigte, von einer Verlängerung der Dauer der Einzelschwankung her, ebenso wie ich dies zuerst für den Froschischiadicus fand: übrigens hat bereits Fuchs eine solche, im Laufe des Rheotomversuchs sich einstellende "Nachwirkung" andeutungsweise konstatiert.

Freilich fand sich oft auch progressive Abnahme der Grösse der integralen negativen Schwankung, wie auch des Maximums der Rheotomkurve im Laufe der Versuche: bei solchen Präparaten fand 
aber diese Abnahme auch ohne bäufige Reizung statt, zum Zeichen, dass es sich nicht um Ermüdung, sondern um das oben schon erwähnte rasche $A b$ sterben handelte.

Eine Vergrösserung der integralen negativen Schwankung des Demarkationsstroms ist auch beim marklosen Kephalopodennerven die Folge der ersten Einwirkung eines mässigen Kohlensäurestroms sowie der Narkose mit Äther-oder Chloroformdampf: bei Fortdauer der Einwirkung nimmt die negative

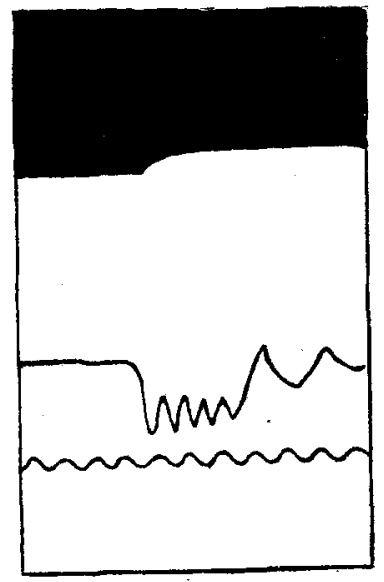

a.

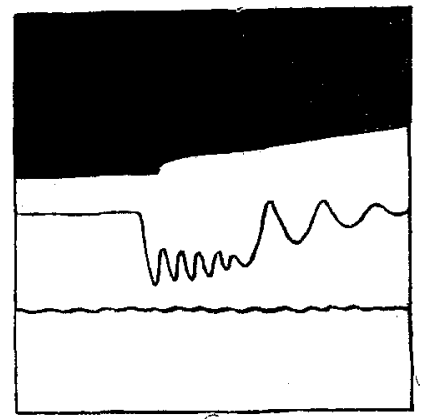

b.

Fig. $1 a$ und $b$. Einzelaktionsstrom eines Octopusnerven auf Öfnungsinduktionsschlag ${ }^{1}$ ): $a$ normal; $b$ Kohlensäurenachwirkung. Zeit $=1 / 50$ Sek.

Schwankung ab bis zum Verschwinden, um nach Abstellung des Kohlensäure- resp. Ätherdampfstroms wieder zu erscheinen, und zwar bei gut überlebenden Nerven stets verstärkt gegen den Anfangswert: sowohl die initiale Verstärkung wie diejenige nach Abstellung des chemischen Agens erwies sich durch die Rheotom- und Kapillarelektrometeranalyse als bedingt durch die Verlängerung des absteigenden Schenkels der Aktionsstromskurve oder sog. "negative Nachwirkung", ganz wie beim markhaltigen Froschnerven, wie aus den angehängten Protokollen der Rheotomversuche ersichtlich ist sowie aus Figur 1, wo $a$ den Einzelaktionsstrom des normalen Octopusmantelnerven, $b$ denselben in der

1) Der Reizsignalstrom öffnete bei seiner Schliessung durch ein Relais den primären Strom des Induktoriums, und umgekehrt! 
"Kohiensäurenachwirkung" darstellt, - aufgenommen mit Registrierung der Kapillarelektrometerbewegung.

In anderen Fällen freilich erschien nach Abstellen der Kohlensäure oder des Narkotikums die galvanometrisch beobachtete negative Schwankung nur geschwächt wieder, um dann immer weiter abzunehmen: es waren das eben immer solche Nerven, deren Erregbarkeit resp. Grösse der negativen Schwankung auch ohne jede Behandlung ständig abnahm, die also rasch abstarben.

Im übrigen möchte ich bemerken, dass ich beim Octopusnerven auch nach Einwirkung von $\mathrm{Chl}$ or of orm rasche und vollständige Erholung gesehen habe, und überhaupt den Eindruck empfing, als ob dieses Objekt gegen die flüchtigen Narkotika relativ starke Resistenz zeige, im Gegensatz zu der gleich zu besprechenden Erstickung. Wie weit diese Resistenz etwa durch den geringen (ja nicht ganz fehlenden) Myelingehalt bedingt sei, sowie auf sonstige theoretische Erörterungen möchte ich hier nicht eingehen.

Über die Erstickung des Octopusnerven habe ich zwei Versuche angestellt; der Stickstoff wurde durch Erhitzen von Kaliumnitrit und Chlorammonium dargestellt und durch Schütteln in einem Gasometer und Durchleiten durch mehrere Waschflaschen mit der durch v. Baeyer angewendeten alkalischen Lösung von weinsaurem Eisenoxydul gereinigt: die negative Schwankung wurde immer kleiner, um nach etwa $1^{1 / 2}$ Stunde fast völlig zu verschwinden; nach Durchtreiben eines Luftstroms trat in einigen Minuten völlige Erholung ein (siehe Versuchsprotokoll Nr. 20).

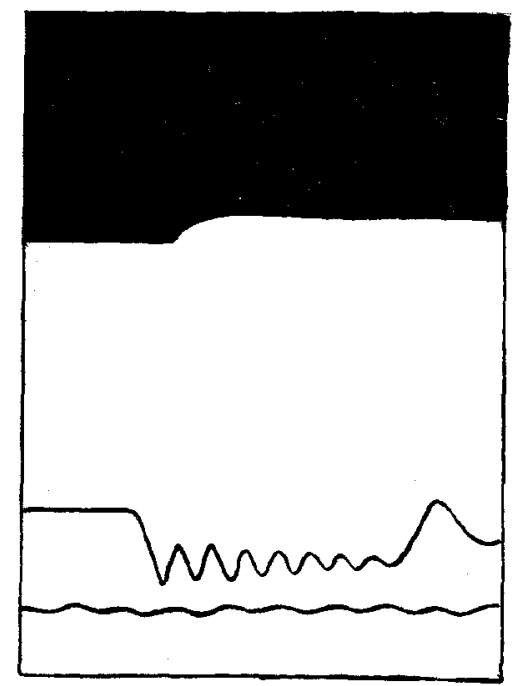

Fig. 2. Wie Fig. 1 a, nur grössere Plattengeschwindigkeit.

Es galt nun, auch an dem vorliegenden Objekt, das elektrische Verbalten bei der Tätigkeit für eine innerhalb der narkotisierten oder erstickten Strecke und eine jenseits derselben gelegene unbeeinflusste Stelle zu vergleichen; natürlich hat dies gerade an diesem Objekt seine besonderen Schwierigkeiten. Fast stets war die 
galvanometrische, integrale negative Schwankung an der entfernter von der Reizstelle gelegenen Längsschnittelektrode $b$ viel kleiner als an der näher gelegenen $a$, also von vornherein ein beträchtliches Dekrement vorhanden. Bei stärkerer Narkose verschwand gewöhnlich die negative Schwankung in $b$ recht bald völlig, während sie in a noch wenig herabgesetzt war: ein Zeichen dafür, dass auch hier, wie beim markhaltigen Nerven, die Narkose schon für sich ein Dekrement der Erregungswelle hervorruft, hier also ein schon Bestehendes wesentlich verstärkt. Wir dürfen wohl weiter annehmen, dass auch hier wie dort das Dekrement mit der Stärke der Erregung und der Länge der narkotisierten Strecke zunehmen dürfte. Das weitere allgemeine Gesetz, welches die Veränderungen der Erregungswelle durch physikalische wie auch chemische Agentien betriff, konnte ich trotz der in dem starken Dekrement liegenden Schwierigkeiten mehrmals direkt experimentell bestätigen; in einem Stadium, wo die Dauer des einphasischen Aktionsstroms an der proximalen, innerhalb der Gaskammer gelegenen Ableitungsstelle $a$ stark verlängert war, in Gestalt einer rheotomisch erkennbaren sog. negativen Nachwirkung, war an der distalen, jenseits der Gaskammer gelegenen Ableitungsstelle $b$ davon keine Spur vorhanden. Es gilt also bestimmt das "Lokalisationsgesetz", das ich ja auch am Muskel bestätigt fand, und das ganz offenbar für den Leitungsvorgang in allen erregungsleitenden lebenden Gebilden Gültigkeit hat (siehe Protokolle Nr. 7 und 13).

Anhangsweise möchte ich darüber berichten, dass ich die sich darbietenden Gelegenheiten benutzte, auch noch an einigen an deren Objekten von Seetieren elektrophysiologische Beobachtungen anzustellen.

So präparierte ich an einem besonders grossen Exemplar von Sepia beiderseits das Stellarganglion mit zu- und abführenden Nerven heraus: die ersteren, die eigentlichen Mantelnerven, sind hier, im Gegensatz zu den Polypen, von so kurzem Verlaufe, dass sie für die Anbringung mehrerer Elektroden nicht genügend Platz bieten; dafür sind die strahlenförmig von dem Ganglion ausgehenden Äste um so bedeutender, und an einem derartigen, etwa $20 \mathrm{~mm}$ langen Objekt, habe ich denn auch den Demarkationsstrom und dessen negative Schwankung schon bei recht schwacher Induktionsreizung beobachten, ja selbst einen Rheotomversuch anstellen können, 
welcher eine dem Verhalten der Polypen durchaus analoge Dauer und Fortpflanzungsgeschwindigkeit der Negativitätswelle ergab (siehe Versuchsprotokoll Nr. 8).

Für den elektrischen Nerven des Zitterrochens hat S. Fuchs 1894 angegeben ${ }^{\mathbf{1}}$, dass die elektromotorische Kraft des Demarkationsstroms ganz besonders hoch sei, und dass die negative Schwankung desselben auf mechanische Einzelreizung (Schnitt, Quetschung) sehr deutlich $7 u$ beobachten sei; freilich fügt er gleich hinzu, dass bei Wiederholung der Reizung die Grösse der Schwankung sehr rasch abnehme. Ich kann dieses letztere nun auch für die elektrische Reizung auf das allerentschiedenste bestätigen: am frisch herausgeschnittenen Torpedonerven war zunächst auf einen einzelnen Induktionsschlag hin eine deutliche, bei dem hochempfindlichen Galvanometer bis zu 20 Skalenteile betragende negative Schwankung des sehr kräftigen Demarkationsstroms zu beobachten, sehr bald aber nahm sie ab und war nach wenigen Minuten selbst auf kräftige Tetanisation hin nur noch ganz unbedeutend, so dass nicht einmal die Rheotomanalyse gelang: das kapillarelektrometrische Bild der Schwankung am frischen Nerven zeigt Figur 3. Um

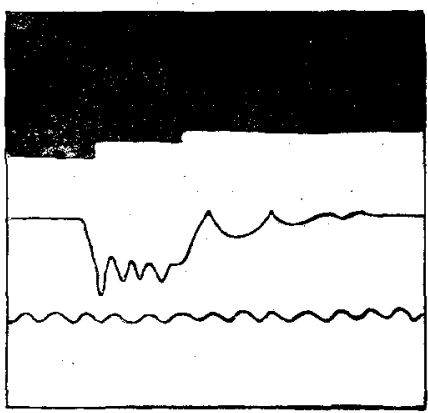

Fig. 3. Einzelaktionsströme eines Torpedonerven auf Öfnungs- und Schliessungsinduktionsschlag. Zeit $\Longrightarrow 1 / 50$ Sek. weiterhin noch sichtbare Aktionsströme zu erhalten, muss der Reiz immer verstärkt werden, und man gelangt zu Rollenabständen, wo ausser Stromschleifen sich der extrapolare Elektrotonus einmischt, welcher an diesem Objekt durchaus typisch ist. An einem "dritten elektrischen Nerven" einer kräftigen Torpedo marmorata betrug bei einer Länge der durchströmten Strecke . . . $=8 \mathrm{~mm}$, Zwischenstrecke . . . . . $=18 \mathrm{~mm}$, abgeleiteten Strecke. . . . . $=15 \mathrm{~mm}$

mit vier Leclanchéelementen

$$
\begin{aligned}
& \text { bei } 10 \Omega \text { Nebenschliessung } A+82 \text { Skalenteile, } \\
& K-62
\end{aligned}
$$

1) Centralbl. f. Physiol. 1894 S. 530. 


$$
\begin{aligned}
& \text { bei } 150 \Omega \text { Nebenschliessung } A+120 \text { Skalenteile, } \\
& K-90 \quad, \\
& \text { und abgeleiteten Strecke von } 10 \mathrm{~mm} \\
& \text { bei } 10 \Omega \text { Nebenschliessung } A+61 \text { Skalenteile, } \\
& K-45 \quad " \\
& \text { " } 150 " \quad \text { " } \quad \text { " } \\
& K-80 \quad \text { " }
\end{aligned}
$$

Kapillarelektrometerphotogramme zeigen deutlich den Beginn der negativen Einzelschwankung nach demjenigen des Elektrotonus, ähnlich wie in den Garten'schen Versuchen am Hechtolfactorius.

Die rasche Abnahme der negativen Schwankung erwies sich als unabhängig von Reizungsversuchen: es handelt sich also nicht um Ermüdung, sondern um schnelles Absterben, wahrscheinlich infolge Unterbrechung der hier anscheinend sehr reichlichen Blutversorgung. An Versuche über Narkose oder Erstickung der Torpedonerven war natürlich unter solchen Umständen nicht zu denken: möglich, dass sich einmal ein Kunstgriff zur längeren Überlebenderhaltung dieses interessanten Objektes findet.

Endlich habe ich, um auch marklose Nerven von Seefischen in den Kreis der Untersuchung zu ziehen, den N. olfactorius vom Seeaal, Muraena Conger, untersucht: bei grossen Individuen bildet derselbe ein nicht minder ansehnliches Objekt als derjenige des Hechtes, auch ist seine anatomische Lage und Präparationsmethode genau entsprechend.

Der Längsquerschnittsstrom ist hier nun ausserordentlich beträchtlich; die extrapolaren elektrotonischen Ströme schwach, aber deutlich, $-A$ beträchtlich grösser als $K-$; zu meinem Erstaunen gelang es mir aber nicht, auch nur eine Spur von negativer Schwankung zu erhalten, weder bei Induktionsreizen (Einzelschlägen oder Tetanisieren) noch beim Öffnen und Schliessen des konstanten Stroms, obwohl kräftige Tiere vorlagen und die Präparation so schnell wie möglich nach Abtrennung des Kopfes erfolgte, bei noch sehr starkem Tonus der Muskulatur. Die Elektrophysiologie und Elektropathologie der Fischnerven scheint also noch manche Schwierigkeiten und Überraschungen zu bieten.

Die Ausführung dieser Arbeit in Arcachon wurde durch ein von dem Kuratorium gütigst bewilligtes Reisestipendium aus der Gräfin-Bose-Stiftung ermöglicht; für Anschaffung und Trans- 
port der Apparate, ebenso wie für die Kosten der demnächst zu berichtenden Versuche über die Elektropathologie der Warmblüternerven kam ferner eine Geldbewilligung der kgl. Gesellschaft der Wissenschaften zu Göttingen sehr zu statten. Für beides sei an dieser Stelle der allerverbindlichste Dank ausgesprochen.

\section{Versuchsprotokoll Nr. 7.}

Beide Mantelnerven eines grossen Octopus zusammengelegt. Reizelektroden r $r$; Längsschnittableitung $a$ in der Gaskammer; $b$ jenseits derselben; Entfernungen $r a=20 \mathrm{~mm}, a b=20 \mathrm{~mm}$; von $b$ bis zum Querschnitt $q=4 \mathrm{~mm}$.

$10^{\mathrm{b}}$ Längsquerschnittstrom $a q \ldots \ldots . . . . .>1000$ Skalenteile Bei Tetanisation (R.-A. $20 \mathrm{~cm}$ ) negative

Schwankung

bei $a \ldots \ldots \ldots \ldots$. . . . . -190 "

bei $b \ldots \ldots \ldots \ldots \ldots \ldots$. 112 ,

$10 \mathrm{~h} 5^{\prime}$ Rheotomversuch: 1 Schieberstrich $=0,001$ Selsunde. Kontaktzeit von $95-97 . \%$

Ableitung bei $a$ :

Schieberstrich Ablenkung

$\begin{array}{rl}0 & - \text { Spur } \\ 1 & -3 \\ 2 & -9 \\ 4 & -3 \\ 15 & 0\end{array}$

Ableitung bei $b$ :

Schieberstrich Ablenkung

$\begin{array}{rr}3 & 0 \\ 5 & 0 \\ 7 & -2 \\ 9 & -5 \\ 11 & -3 \\ 18 & 0\end{array}$

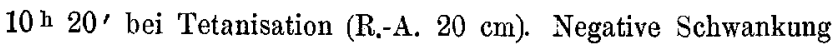

bei $a \ldots \ldots$. . . . . . . . . . . . . . -182 Skalenteile

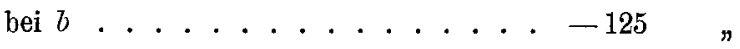

$10^{\text {h }} 30^{\prime}$ desgleichen

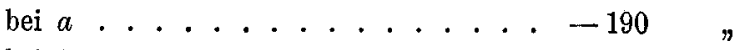

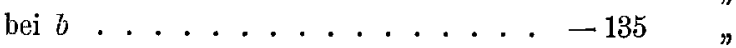

$10^{\mathrm{h}} 35^{\prime}$ Kohlensäure in mässigem Strom eingeleitet.

$10^{\mathrm{h}} 40^{\prime}$ bei Tetanisation (R.-A, $20 \mathrm{~cm}$ ). Negat. Schwankung

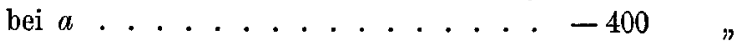

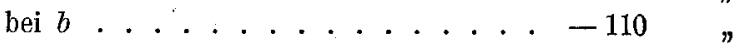

10 h 48 ' Längsquerschnittstrom $a q \ldots . . . .+750$. . .

bei Tetanisation (R.-A. $20 \mathrm{~cm}$ )

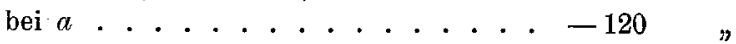

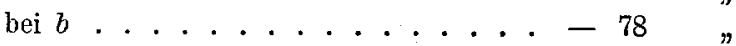

$10^{\mathrm{h}} 50^{\prime}$ Kohlensäurestrom abgestellt, Luft durchgeleitet.

$10^{\text {h }} 52^{\prime}$ bei Tetanisation (R.-A. $20 \mathrm{~cm}$ ). Negat. Schwankung

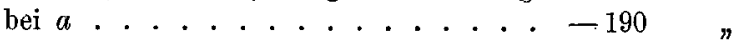

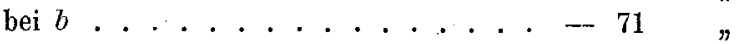


10 h 54 ' Rheotomversuch (Tourenzahl und Kontaktzeit. wie oben).

Ableitung bei $a$ :

Schieberstrich Ablenkung

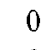

2

15

40
$-2$

$-9$

$-4$

$-3$
Ableitung bei $b$ :

Schieberstrich Ablenkung

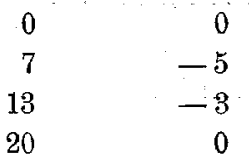

$11^{\text {h }}$ Längsquerschnittstrom $\alpha q \ldots$. . . . . . . + +800 Skalenteile

Bei Tetanisation (R.-A. $20 \mathrm{~cm}$ ). Negative

Schwankung

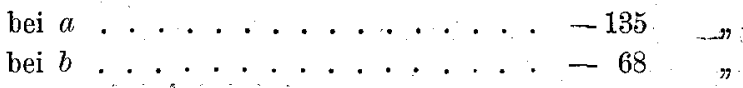

Versuchsprotokoll Nr. 13.

Beide Mantelnerven eines Octopus. Gleiche Anordnung wie bei Protokoll Nr. 7. Distanzen $r a=12 \mathrm{~mm}, a b=15 \mathrm{~mm}, b q=3 \mathrm{~mm}$.

1 h $30^{\prime}$ Längsquerschnittstrom $a q \ldots \ldots+250$ Skalenteile $b q \ldots \ldots+230$.....

Bei Tetanisation (R.-A. $23 \mathrm{~cm}$ ). Negat. Schwankung

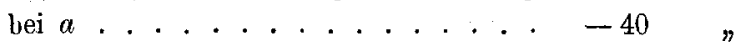

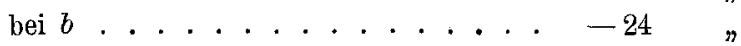

1 h $40^{\prime}$ Rheotomversuch (hier nicht näher mitgeteilt).

$1^{\mathrm{h}} 58^{\prime}$ bei Tetanisation (R.-A. $23 \mathrm{~cm}$ ). Negat. Schwankung

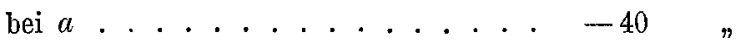

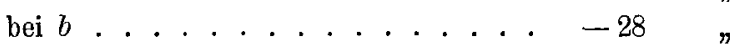

$2 \mathrm{~h}$ Ätherdampf eingeleitet.

$2 \mathrm{~h} 7 \cdot$ bei Tetanisation (R.-A. $23 \mathrm{~cm}$ ). Negat. Schwankung

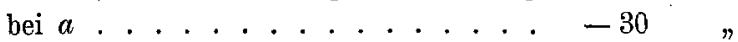

bei $b \ldots \ldots \ldots \ldots \ldots$

2 h $15^{\prime}$ desgleichen. Negative Schwankung

bei $a \ldots \ldots$. . . . . . : : . . . . . . -24

bei $b$. . . . . . . . . . . . . . -12 "

2 h 17' Längsquerschnittstrom $a q$. . . . . . . . + + 50 " "

$b q . \ldots . . .+111 \%$

$2^{\text {h }} 20^{\prime}$ Äther abgestellt, Luft durchgeleitet.

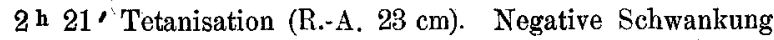

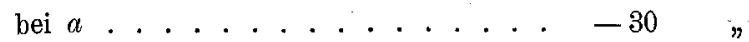

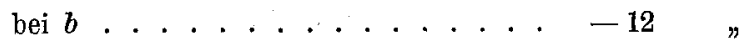

2 h 22' Tetanisation (R.-A. $23 \mathrm{~cm}$ ). Negative Schwankung

bei $a \ldots \ldots$.............

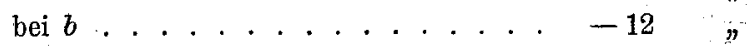

Desgleichen (R.-A. $20 \mathrm{~cm})$. Negat. Schwankung

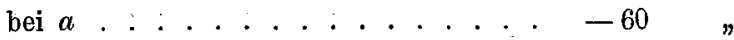

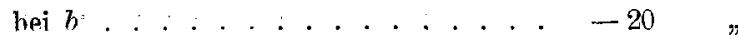


2 h 39 ' desgleichen (R.-A. $23 \mathrm{~cm}$ ). Negative Schwankung bei $a \ldots \ldots \ldots \ldots$. . . . . . -29 Skalenteile bei $b \ldots \ldots \ldots \ldots$

Desgleichen (R.-A. $20 \mathrm{~cm}$ ). Negat. Schwankung bei $a \ldots \ldots \ldots \ldots$ bei $b . . . . . . . . .26$. .

$2^{\mathrm{h}} 40^{\prime}$ Längsquerschnittstrom $a q$ und $b q \ldots+135$. . . .

\section{Versuchsprotokoll Nr. 20.}

Einfacher Octopusmantelnerv. Die nämliche Anordnung. Distanzen $r^{r} \alpha=30 \mathrm{~mm}$, $a b=15 \mathrm{~mm}, b q=10 \mathrm{~mm}$.

9 h 59' Längsquerschnittstrom $a q$. . . . . . . . + 200 Skalenteile

$$
\text { bq......... + }+500 \text { " }
$$

$10^{\mathrm{h}} 2^{\prime}$ Tetanisation (R.-A. $17 \mathrm{~cm}$ ). Negative Schwankung

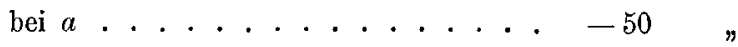

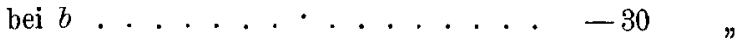

$10 \mathrm{~h} 07$ ' Stickstoff durchgeleitet.

$10 \mathrm{~h} 12$ ' Tetanisation (R.-A. $17 \mathrm{~cm}$ ). Negative Schwankung

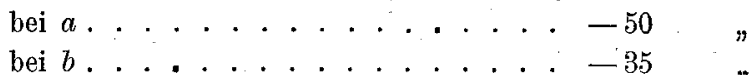

10 27 ' desgleichen (R.-A. $17 \mathrm{~cm}$ ), Negative Schwankung bei a.............. -26 "

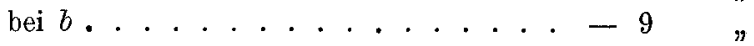

10 b $28^{\prime}$ desgleichen (R.-A. $17 \mathrm{~cm}$ ). Negative Schwankung bei $a \ldots \ldots \ldots \ldots$ bei $b \ldots \ldots \ldots$............ 0

desgleichen (R.-A. $10 \mathrm{~cm}$ ) (Anelektrotonus ?) bei $a . \ldots . . . . . . .+5 n$ n bei $b \ldots \ldots . \ldots . \ldots$

$10^{\text {h }} 39^{\prime}$ Stickstoff abgestellt, Luft zugelassen.

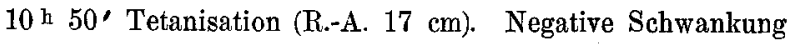

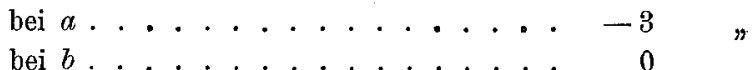

$10^{\mathrm{h}} 48^{\prime}$ desgleichen (R.-A. $17 \mathrm{~cm}$ ). Negative Schwankung bei $a . . . . . . . . . . .4-25 n$

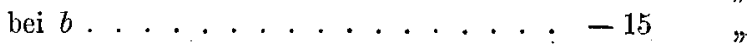

$10 \mathrm{~h} 57$ ' desgleichen (R.-A. $17 \mathrm{~cm}$ ). Negative Schwankung

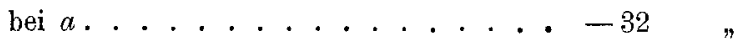

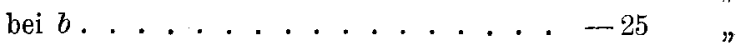
Desgleichen (R.-A. $7 \mathrm{~cm})$

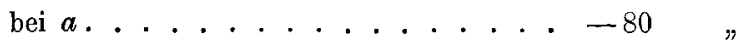

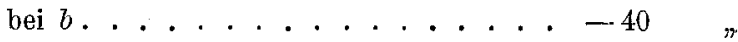




\section{Versuchsprotokoll Nr. 8.}

Sternganglionnerv einer grossen Sepia. Abstand zwischen den Reizelektroden und der Längsschnittelektrode $r l=5 \mathrm{~mm}$; zwischen der Längs- und der Querschnittelektrode $l q=10 \mathrm{~mm}$.

11 h Längsquerschnittstrom ............ + + 168 Skalenteile

Bei Tetanisation (mit $23 \mathrm{~cm} \mathrm{R}$-A.). Negative

Schwankung ........... . . -40 "

Desgleichen (mit $20 \mathrm{~cm} \mathrm{R.-A.).} \mathrm{Negat.} \mathrm{Schwankung}-70 "$

11 h $20^{\prime}$ Rheotomversuch (1 Schieberstrich $=0,001$ Sekunde), Kontaktzeit von von 95 bis 97 .

$\begin{array}{cc}\text { Schieberstrich } & \text { Ablenkung } \\ 1 & 0 \\ 1,5 & - \text { Spur } \\ 2 & -2 \\ 3 & -8 \\ 4 & -7 \\ 6 & -3 \\ 10 & -0\end{array}$

Fortpflanzungszeit von 95 bis $1,5=0,0065$ Sekunden. Weg $=5 \mathrm{~mm}$, ergibt $v \neq 1,3 \mathrm{~m}$.

$1 \mathrm{~h} 50^{\prime}$ bei Tetanisation (mit $23 \mathrm{~cm} \mathrm{R.-A.).} \mathrm{Negat.} \mathrm{Schwankung}-58$ Skalenteile

Desgleichen (mit $20 \mathrm{~cm} \mathrm{R.-A.).} \mathrm{Negat.} \mathrm{Schwankung}-90$ " 